\title{
CONSUMO DE BEBIDAS ALCOÓLICAS POR ADOLESCENTES: problemas relacionados e fatores associados
}

\author{
Kelly Oliva JORGE ${ }^{1}$ \\ Anderson OLIVEIRA ${ }^{2}$ \\ Samyra Maria dos Santos Nassif LACERDA ${ }^{3}$ \\ Sérgio Ricardo MAGALHÃES ${ }^{4}$ \\ Soraya de Mattos Camargo GROSSMANN ${ }^{5}$ \\ Camilo Aquino MELGAÇO 6
}

\begin{abstract}
${ }^{1}$ Doutora em Odontopediaria. Docente do curso de Odontologia da Universidade Vale do Rio Verde - UninCor, campus Belo Horizonte. E-mail: kellyoliva@yahoo.com.br

${ }^{2}$ Acadêmico do curso de Odontologia da Universidade Vale do Rio Verde - UninCor, campus Belo Horizonte. E-mail: andersonodontologia @ outlook.com

${ }^{3}$ Doutora em Biologia Celular. Docente do curso de Odontologia da Universidade Vale do Rio Verde - UninCor, campus Belo Horizonte. E-mail: samyranassif@ hotmail.com

${ }^{4}$ Doutor em Engenharia Biomédica. Docente do curso de Odontologia da Universidade Vale do Rio Verde - UninCor, campus Belo Horizonte. E-mail: sergio.magalhaes@unincor.edu.br

${ }^{5}$ Doutora em Patologia Bucal. Docente do curso de Odontologia da Universidade Vale do Rio Verde - UninCor, campus Belo Horizonte. E-mail: prof.soraya.grossmann@unincor.edu.br

${ }^{6}$ Doutor em Ortodontia. Docente do curso de Odontologia da Universidade Vale do Rio Verde - UninCor, campus Belo Horizonte. E-mail: camilomelgaco@ hotmail.com
\end{abstract}

Recebido em: 13/12/2016 - Aprovado em: 26/05/2017 - Disponibilizado em: 01/07/2017

\section{RESUMO:}

Buscou-se avaliar o consumo de álcool e problemas relacionados entre adolescentes, e sua associação com gênero, fatores socioeconômicos, religiosidade e comportamento dos pais em relação ao hábito de beber. O estudo transversal contou com uma amostra de conveniência de 436 adolescentes de 17 a 19 anos de idade, matriculados em escolas públicas e privadas da cidade de Belo Horizonte. Realizou-se a análise estatística descritiva e bivariada. A prevalência do consumo de bebidas alcoólicas foi de 71,8\%. A incapacidade de lembrar algo após o consumo de bebida alcoólica foi a consequência mais relatada após o consumo de álcool pelos participantes (20.2\%). Houve associação estatisticamente significante entre o consumo de bebidas alcoólicas e aqueles adolescentes com melhores condições socioeconômicas, filhos de pais que fazem o uso do álcool e adolescentes que frequentam com menor frequência a igreja. O sexo não foi estatisticamente associado ao consumo de bebidas alcoólicas. A identificação do consumo de álcool pelo adolescente, bem como dos problemas relatados é condição extremamente relevante para preveni-los de forma eficaz. Ampliar a discussão sobre o consumo de bebidas alcoólicas e fatores de risco relacionados poderá fornecer ferramentas para o planejamento de estratégias que busquem a promoção da saúde e qualidade de vida destes adolescentes de acordo com seus respectivos contextos sociais.

Palavras chave: Bebidas Alcoólicas. Adolescentes. Odontologia. Fatores socioeconômicos. Religiosidade.

\section{ALCOHOLIC BEVERAGE CONSUMPTION BY ADOLESCENTS: related problems and associated factors}

\begin{abstract}
:
This study aimed to evaluate the alcohol consumption and problems related between adolescents, and its association with socioeconomic factors, religiosity and behavior of the parents in relation to the alcoholic beverage consumption. This transversal study was based on a sample of convenience of 436 adolescents between 17 and 19 years of age, from
\end{abstract}


public and private schools of the city of Belo Horizonte. A descriptive and bivariate analysis was conducted. The prevalence of the alcoholic beverage consumption was of $71,8 \%$. The incapacity to remember something after alcoholic beverage consumption was the most related aspect by the participants $(20,2 \%)$. There was statistical significant association between the alcoholic beverage consumption and those adolescents with better socioeconomic conditions, children of parents who make the use of alcohol and adolescents less frequent to the church. Gender was not associated with the alcoholic beverage consumption. The identification of the alcohol consumption habit and its associated problems is a relevant manner to prevent these problems. Extending the discussion about the alcoholic beverage consumption and related factors of risk will provide tools for planning strategies aiming the promotion of health and quality of life of these adolescents in their respective social contexts.

Keywords: Alcoholic beverages, Adolescents, Dentistry, Socioeconomic factors, Religiosity.

\section{INTRODUÇÃO}

A adolescência é uma etapa da vida na qual muitas vezes há experimentação de drogas, sejam elas lícitas ou ilícitas. Embora na maioria das vezes esse uso seja apenas experimental, é possível notar comportamentos que refletem esses padrões na vida adulta (TAVARES et al., 2001).

O encorajamento proporcionado pelo consumo de bebidas alcoólicas acaba por ser um atrativo característico nesta idade de descobrimento da vida. $\mathrm{O}$ excesso do consumo de álcool pelos adolescentes além de encorajá-los a assumirem comportamentos de risco, muitas vezes é um fator considerado pelo grupo ao que o adolescente frequenta, e isso, pode the inserir como membro ativo do grupo ou não (ROMANO et al., 2007).

Dentre os fatores associados ao consumo de bebidas alcoólicas, o fator socioeconômico se destaca, como observado por Martins-Oliveira et al. (2015), onde adolescentes que vivem em uma área de baixa vulnerabilidade social são mais propensos a consumir álcool em comparação com aqueles que tem piores condições socioeconômicas. A família, instituidora das relações primárias dos seus membros, influencia a forma como o adolescente reage à ampla oferta de drogas, lícitas ou ilícitas, na sociedade atual. Relações familiares saudáveis desde o nascimento da criança servem como fator de proteção para toda a vida e, de forma muito particular, para o adolescente (POZZA et al., 2011).

Segundo estudo de Delma et al. (2005) numa investigação com relação ao uso de álcool por adolescentes escolares de escolas públicas no Brasil, há mais de duas décadas o álcool ocupa o primeiro lugar de consumo entre os estudantes da rede estadual de ensino, seguido à distância por outras substâncias. Neste estudo, realizado em dez capitais brasileiras, pode-se observar um discreto predomínio do uso de álcool pelo sexo masculino, com início precoce entre 10 a 12 anos de idade.

A religiosidade e o consumo de álcool e drogas ilícitas tem sido objeto de estudo de muitos pesquisadores, pois, para o adolescente, ambos são fatores muito significativos para sua experiência pessoal e social (DALGALARRONDO et al., 2004). Estudos mostram que os aspectos religiosos apresentados aos adolescentes no ambiente 
familiar podem conscientizar o mesmo sobre o consumo de bebidas alcoólicas, diminuindo assim os possíveis efeitos reflexos ao uso em excesso de álcool. Adolescentes que exibem uma vida religiosa ativa e participante tendem a consumir o álcool esporadicamente ou mesmo nem consumir (CASTRO et al., 2012). As evidências apresentadas na literatura sugerem que a religiosidade e a espiritualidade são fatores associados à saúde e ao bem-estar em adolescentes. Isso se faz pela presença dos conceitos de certo ou errado e do bem e mal apresentados pela igreja, levando o indivíduo a ter uma visão mais clara dos efeitos do uso abusivo de álcool na sua vida, não apenas na adolescência, mas também na vida adulta (BEZERRA et al., 2009). O consumo de bebidas alcoólicas por adolescentes pode resultar em implicações negativas em sua vida, como a diminuição dos níveis de escolaridade, exposição dos mesmos a acidentes de trânsito, envolvimento com o sistema de justiça criminal, brigas e internações hospitalares (HUMENSSKY, 2010). Também, como possível consequência ao consumo excessivo de álcool pelos adolescentes podemos destacar o traumatismo dentário. A associação entre o consumo de álcool e a violência interpessoal é bem reconhecida, e esta relação está cada vez mais estreita. Homens jovens com média de idade de 16-20 anos representam um grupo da população com grandes chances de sofrerem algum tipo de dano decorrente da violência física quando sob a influência do álcool, sendo as lacerações e fraturas faciais as situações emergenciais mais prevalentes (LAVERICK et al., 2008), isto por ser a região de cabeça e pescoço a área anatômica mais favoravelmente atingida pelos atos de violência (EL-MAAYTAH et al., 2008).

A identificação do consumo de álcool pelo adolescente, bem como dos problemas relatados é condição extremamente relevante para preveni-los de forma eficaz. Ampliar a discussão sobre o consumo de bebidas alcoólicas e dos fatores de risco relacionados poderá fornecer ferramentas para o planejamento de estratégias que busquem a promoção da saúde e qualidade de vida destes adolescentes de acordo com seus respectivos contextos sociais.

Assim, foi objetivo do presente estudo avaliar a prevalência do consumo de bebidas alcoólicas e problemas relacionados entre adolescentes da cidade de Belo Horizonte, Minas Gerais/Brasil; e determinar associação com gênero, fatores socioeconômicos, religiosidade e comportamento dos pais em relação ao consumo do álcool.

\section{METODOLOGIA}

Foi desenvolvido um estudo transversal, com uma amostra de conveniência de 436 adolescentes de 17 a 19 anos, matriculados em escolas das redes pública e privada da cidade de Belo Horizonte. A coleta de dados foi realizada no ano de 2012. Com base na consulta de listas fornecidas pela Secretaria Municipal de 
Educação e Secretaria Estadual de Educação de Minas Gerais, 18 escolas pertencentes a cada regional político-administrativa da cidade (Barreiro, Centro-sul, Leste, Nordeste, Noroeste, Norte, Oeste, Pampulha e Venda Nova) foram sorteadas para compor o estudo. Para cada regional político-administrativa de Belo Horizonte foram sorteadas duas escolas, uma da rede pública (escolas municipais e estaduais) e uma da rede privada. Foram considerados como critérios de elegibilidade escolares de ambos os gêneros, sem limitações cognitivas ou físicas que impossibilitassem a compreensão ou o preenchimento dos questionários, uma vez identificados pelas respectivas professoras em sala de aula antes da entrega dos questionários.

\section{Instrumentos}

O Teste para Identificação de Problemas Relacionados ao Uso do Álcool (AUDIT) foi utilizado para a avaliação do consumo de bebidas alcoólicas. O AUDIT é um questionário auto-respondido, validado no Brasil (LIMA et al., 2005), composto por dez questões e as respostas a cada questão são pontuadas de 0 a 4. Classifica-se o usuário em uma de quatro zonas de risco de acordo com o escore obtido: zona I: 0-7 indica uso de baixo risco ou abstinência; zona II: 8-15 indica uso de risco; zona III: 16-19 sugere uso nocivo e zona IV: $\geq 20$ mostra uma possível dependência, (MAGNABOSCO et al., 2007).
O questionário foi aplicado em um estudo piloto, não demonstrando necessidade de mudanças. O estudo piloto foi conduzido com uma amostra de conveniência de 101 adolescentes, de ambos os gêneros, com idade entre 15 a 19 anos, matriculados e aleatoriamente selecionados de uma escola pública e uma privada de uma regional político-administrativa de Belo Horizonte, e não fizeram parte da amostra principal.

Os dados pessoais e sociodemográficos como gênero, idade, endereço e escolaridade dos pais; e sobre religiosidade e uso de bebidas alcoólicas pelos familiares foram coletados seguindo uma ficha entregue aos adolescentes posteriormente ao preenchimento do questionário.

A participação religiosa pelos adolescentes e o consumo de bebidas alcoólicas pelos pais foram avaliados através das seguintes questões: “a) Você tem religião (sim/não)? Qual?; b) Com que frequência você vai à igreja (mensalmente/nunca; diariamente/semanalmente)?; c) Seu pai consome bebidas alcoólicas (sim/não)? E sua mãe (sim/não)?" (BARTKOWSKI e XU, 2007; SANCHEZ et al., 2011).

Como critérios de classificação socioeconômica foram utilizados o tipo de escola a qual o adolescente pertencia (pública ou privada) e a escolaridade da mãe, correspondente aos anos de estudo formais relatados pelos participantes. A escolaridade materna foi dicotomizada pela mediana. 


\section{Considerações éticas}

Este estudo foi aprovado pelo Comitê de Ética em Pesquisa com Seres Humanos da Universidade Federal de Minas Gerais e contou com a aprovação da Secretaria Municipal de Educação do município (SMEMG) e a Secretaria Estadual de Educação (SEE-MG). Os adolescentes e seus responsáveis assinaram o Termo de Consentimento Livre e Esclarecido. Foi garantido ainda, o direito de não identificação aos participantes.

\section{Análise estatística}

Os dados desta pesquisa foram analisados de forma descritiva e analítica (teste qui-quadrado; $\mathrm{p}<0.05$ ) através do Package for the Social Sciences (SPSS for Windows, versão 20.0, SPSS Inc, Chicago, IL, USA).

\section{RESULTADOS}

A amostra final consistiu em 436 adolescentes com idade entre 17 e 19 anos matriculados em escolas públicas $(n=331$; $75.9 \%)$ e em escolas privadas $(n=105 ; 24.1 \%)$ da cidade de Belo Horizonte, Brasil. Da amostra total, 59,6\% eram do sexo feminino, sendo a maioria dos participantes (50.7\%) filhos de mães com oito ou mais anos de escolaridade. A prevalência do consumo de bebidas alcoólicas foi de 71,8\% ( $\mathrm{n}=313)$, sendo que 94 adolescentes (21.6\%) se encontravam na zona de uso de risco à possível dependência do álcool. Com relação à idade de início do consumo de álcool, $34.2 \%(n=149)$ dos participantes relataram ter iniciado o consumo na faixa etária compreendida entre 10 a 14 anos, apresentados na Tabela 1.

Tabela 1- Distribuição da amostra de acordo com variáveis independentes, Belo Horizonte, Minas Gerais, Brasil, 2012.

\begin{tabular}{l|c}
\hline Masculino & $176(40.4)$ \\
Feminino & $260(59.6)$ \\
\hline Escolaridade da mãe (anos de estudo formal) & $140(32.1)$ \\
\hline 0 a 7 ou mais & $211(50.7)$ \\
Não souberam responder & $75(17.2)$ \\
\hline Consumo de álcool & \\
\hline Abstêmio & $123(28.2)$ \\
Não abstêmio & $313(71.8)$ \\
\hline Risco do consumo de álcool (AUDIT) & $342(78.4)$ \\
\hline$<7$ pontos: uso de baixo risco ou abstinência & $94(21.6)$ \\
$\geq 8$ pontos: uso de risco à possível dependência & \\
\hline Idade de início do consumo de álcool & $149(34.2)$ \\
\hline 10-14 anos & $162(37.1)$ \\
15-18 anos & $125(28.7)$ \\
\hline
\end{tabular}


amostra em relação ao padrão de consumo de

Tabela 2- Distribuição da amostra de acordo com o padrão de consumo de bebidas alcoólicas, Belo Horizonte, Minas Gerais, Brasil, 2012

\begin{tabular}{l|c}
\hline \multicolumn{1}{c|}{ Variáveis Independentes } & Frequência n(\%) \\
\hline Frequência de consumo de álcool & $305(70.0)$ \\
\hline $0-1$ vez por mês & $131(30.0)$ \\
2 vezes por mês ou quase todos os dias & $359(82.3)$ \\
\hline Doses de álcool consumidas em dia normal & $77(17.7)$ \\
\hline $0-1$ & $140(60.6)$ \\
2 ou mais & $211(39.4)$ \\
\hline Consumo de 5 doses ou mais em uma única ocasião & \\
\hline Nunca & \\
\hline
\end{tabular}

Em relação às consequências associadas ao consumo do álcool, a maior parte dos participantes relatou a incapacidade de lembrar algo após o consumo de bebida alcoólica $(20.2 \%)$ e o sentimento de culpa ou remorso por ter bebido (16.5\%), conforme apresentado na Tabela 3.

Tabela 3 - Distribuição da amostra de acordo com as consequências relacionadas ao consumo do álcool, Belo Horizonte, Minas Gerais, 2012

\begin{tabular}{|c|c|c|}
\hline $\begin{array}{c}\text { Consequências relacionadas } \\
\text { ao uso do álcool }\end{array}$ & Categorias & $\begin{array}{l}\text { Frequência } \\
\mathbf{n}(\%)\end{array}$ \\
\hline $\begin{array}{l}\text { Tentativa frustrada ao tentar } \\
\text { parar de beber }\end{array}$ & $\begin{array}{l}\text { Nunca } \\
\text { Menos que uma vez por mês a uma vez por mês } \\
\text { Uma vez por semana a quase todos os dias }\end{array}$ & $\begin{array}{l}338(89.0) \\
30(6.9) \\
18(4.1)\end{array}$ \\
\hline $\begin{array}{l}\text { Não conseguir fazer o } \\
\text { esperado }\end{array}$ & $\begin{array}{l}\text { Nunca } \\
\text { Menos que uma vez por mês a uma vez por mês } \\
\text { Uma vez por semana a quase todos os dias }\end{array}$ & $\begin{array}{c}393(90.1) \\
25(5.7) \\
18(4.1)\end{array}$ \\
\hline $\begin{array}{l}\text { Necessidade de beber pela } \\
\text { manhã para se sentir bem }\end{array}$ & $\begin{array}{l}\text { Nunca } \\
\text { Menos que uma vez por mês a uma vez por mês } \\
\text { Uma vez por semana a quase todos os dias }\end{array}$ & $\begin{aligned} & 412(94.4) \\
& 12(2.8) \\
& 12(2.8) \\
&\end{aligned}$ \\
\hline $\begin{array}{l}\text { Culpa ou remorso por ter } \\
\text { bebido }\end{array}$ & $\begin{array}{l}\text { Nunca } \\
\text { Menos que uma vez por mês a uma vez por mês } \\
\text { Uma vez por semana a quase todos os dias }\end{array}$ & $\begin{array}{c}364(83.5) \\
39(8.9) \\
33(7.6)\end{array}$ \\
\hline Incapacidade de lembrar algo & $\begin{array}{l}\text { Nunca } \\
\text { Menos que uma vez por mês a uma vez por mês } \\
\text { Uma vez por semana a quase todos os dias }\end{array}$ & $\begin{array}{c}348(79.8) \\
55(12.6) \\
33(7.6) \\
\end{array}$ \\
\hline $\begin{array}{l}\text { Preocupação da família ou } \\
\text { amigos ou sugestão para } \\
\text { parar de beber }\end{array}$ & $\begin{array}{l}\text { Nunca } \\
\text { Sim, mas não no último ano } \\
\text { Sim, durante o último ano }\end{array}$ & $\begin{array}{c}378(86.7) \\
16(3.7) \\
42(9.6) \\
\end{array}$ \\
\hline $\begin{array}{l}\text { Ferimento ou prejuízo a você } \\
\text { ou a outros }\end{array}$ & $\begin{array}{l}\text { Nunca } \\
\text { Sim, mas não no último ano } \\
\text { Sim, durante o último ano }\end{array}$ & $\begin{array}{c}392(89.9) \\
18(4.1) \\
26(6.0)\end{array}$ \\
\hline
\end{tabular}


A tabela 4 mostra o resultado da análise bivariada quanto a variável dependente "Consumo de álcool" e variáveis independentes. Não houve associação estatisticamente associada entre o consumo de bebidas alcoólicas e o gênero $(\mathrm{p}=0.061)$.

Tabela 4 - Resultado da análise bivariada entre consumo de álcool e variáveis independentes - Belo Horizonte, Minas Gerais, 2012

\begin{tabular}{|c|c|c|c|c|}
\hline Variáveis independentes & Amostra total & \multicolumn{2}{|c|}{ Consumo de álcool n(\%) } & $\begin{array}{c}\text { Valor de } \\
\text { p }^{*}\end{array}$ \\
\hline \multicolumn{5}{|l|}{ Gênero } \\
\hline $\begin{array}{l}\text { Masculino } \\
\text { Feminino }\end{array}$ & $\begin{array}{l}176(40.4) \\
260(59.6)\end{array}$ & $\begin{array}{c}135(43.1) \\
41(33.3)\end{array}$ & $\begin{array}{r}178(56.9) \\
82(66.7)\end{array}$ & 0.061 \\
\hline \multicolumn{5}{|l|}{ Tipo de escola } \\
\hline $\begin{array}{l}\text { Pública } \\
\text { Privada }\end{array}$ & $\begin{array}{l}331(75.9) \\
105(24.1) \\
\end{array}$ & $\begin{array}{l}106(86.2) \\
88(28.1)\end{array}$ & $\begin{array}{c}225(71.9) \\
17(13.8) \\
\end{array}$ & 0.002 \\
\hline \multicolumn{5}{|l|}{ Escolaridade materna (anos de estudo formal) } \\
\hline $\begin{array}{l}0-7 \\
\geq 8\end{array}$ & $\begin{array}{l}140(38.8) \\
221(61.2) \\
\end{array}$ & $\begin{array}{l}89(63.6) \\
171(77.4) \\
\end{array}$ & $\begin{array}{l}51(36.4) \\
50(22.6) \\
\end{array}$ & 0.004 \\
\hline \multicolumn{5}{|l|}{ Consumo de álcool pela mãe } \\
\hline $\begin{array}{l}\text { Não } \\
\text { Sim }\end{array}$ & $\begin{array}{l}276(63.3) \\
160(36.7) \\
\end{array}$ & $\begin{array}{l}172(62.3) \\
141(88.1) \\
\end{array}$ & $\begin{array}{c}104(37.7) \\
19(11.9) \\
\end{array}$ & 0.000 \\
\hline \multicolumn{5}{|l|}{ Consumo de álcool pelo pai } \\
\hline $\begin{array}{l}\text { Não } \\
\text { Sim }\end{array}$ & $\begin{array}{l}215(49.3) \\
220(50.7) \\
\end{array}$ & $\begin{array}{l}124(57.6) \\
189(85.9) \\
\end{array}$ & $\begin{array}{l}91(42.4) \\
31(14.1) \\
\end{array}$ & 0.000 \\
\hline \multicolumn{5}{|l|}{ Frequência à igreja } \\
\hline $\begin{array}{l}\text { Uma vez por mês/Nunca } \\
\text { Quase todos os dias/Semanalmente }\end{array}$ & $\begin{array}{l}285(65.4) \\
151(34.6) \\
\end{array}$ & $\begin{array}{l}219(76.8) \\
94(62.2)\end{array}$ & $\begin{array}{l}66(23.2) \\
57(37.8) \\
\end{array}$ & 0.001 \\
\hline \multicolumn{5}{|l|}{ Tipo de religião } \\
\hline $\begin{array}{l}\text { Católica } \\
\text { Evangélica/Islamismo/Testemunhas de Jeová } \\
\text { Espírita }\end{array}$ & $\begin{array}{l}289(72.2) \\
93(23.3) \\
18(4.5)\end{array}$ & $\begin{array}{c}223(77.1) \\
42(45.2) \\
14(77.7)\end{array}$ & $\begin{array}{r}66(22.2) \\
51(54.8) \\
4(22.3)\end{array}$ & 0.000 \\
\hline
\end{tabular}

Houve associação estatística entre o consumo de bebidas alcoólicas e os participantes com melhores condições socioeconômicas quando comparados àqueles com condições socioeconômicas menos favorecidas. O consumo de bebidas alcoólicas pelos pais e uma menor frequência de participação na igreja também estiveram estatisticamente associados ao consumo de álcool.

\section{DISCUSSÃO}

A fase da adolescência é um momento de inserção na sociedade e, por isso, muitas vezes o adolescente usa o álcool como artifício para facilitar o convívio social. A alta prevalência de consumo de álcool observada no presente estudo também é encontrada em diversos países, como nos Estados Unidos, (MALDONADO-DEVINCCI et al., 2010), sendo que, em longo prazo, as consequências negativas do consumo do álcool para a saúde 
podem ser percebidas. No Brasil, na cidade de Florianópolis, Baus et al. (2002) concluíram que foi alta a prevalência no consumo de álcool entre adolescentes escolares do primeiro e segundo graus. $\mathrm{O}$ consumo de álcool na adolescência é um fator muito importante a ser explorado pelos pesquisadores, até por ser nos dias de hoje, considerado um problema de saúde pública. $\mathrm{O}$ consumo de bebidas alcoólicas, por se tratar de uma substância lícita, tende a abrir caminho para o uso de outras substâncias, inclusive ilícitas (ROMANO et al., 2007).

No presente estudo $71,9 \%$ dos adolescentes que estudam em escolas privadas e $77.4 \%$ dos filhos de mães com oito anos ou mais de escolaridade relataram consumir bebidas alcoólicas. Estes resultados apontam para um maior consumo de bebidas alcoólicas entre adolescentes com melhores condições socioeconômicas, corroborando com o estudo de Martins-Oliveira et al. (2015), que mostra que adolescentes que vivem em áreas de baixa vulnerabilidade social são mais propensos ao consumo de álcool em comparação com aqueles com piores condições. Baus et al. (2002) também relata que alunos brasileiros do ensino fundamental e médio com melhores condições socioeconômicas apresentam risco duas vezes maior que alunos com menor nível socioeconômico para o consumo de bebidas alcoólicas. Essa hipótese pode estar relacionada ao acesso as festas e ao poder de compra das bebidas. Guimarães et al. (2004) identificaram uma maior disponibilidade de recursos financeiros (nível socioeconômico e trabalho) como um facilitador para a compra e consumo de bebidas alcoólicas.

Vale ressaltar que a escola frequentada pelos adolescentes além de ser um centro educacional é um ambiente que favorece a socialização e humanização do ser humano. No Brasil, estudantes matriculados na rede pública são tidos como de pior condição socioeconômica. PAIVA et. al. (2014) relatam que as escolas públicas brasileiras são conhecidas por terem menos recursos educacionais do que as privadas, por isso os adolescentes mais ricos no Brasil são matriculados em escolas privadas, evidenciando assim um problema de ordem socioeconômica.

Percebe-se também que o grau de instrução e conhecimento da mãe, dado pelos anos formais de estudo, não minimizou a entrada do álcool na vida de seus filhos. Talvez a presença e participação familiar estejam mais associadas a este fato (MARTINS-OLIVEIRA et al., 2015; POZZA et al., 2011)

Apesar do sexo não ter sido um fator estatisticamente associado ao consumo de bebidas alcoólicas no presente estudo, há pesquisas que demonstram discreto predomínio do consumo de álcool pelo sexo masculino (DELMA et al., 2005; MARTINSOLIVEIRA et al., 2015; ZARZAR et al., 2012). Porém, este padrão de análise segundo o gênero, vem sendo discutido em estudos envolvendo a epidemiologia do consumo de 
bebidas alcoólicas. Nesta análise, se torna importante salientar o consumo de bebidas pelo gênero feminino, dadas as suas conquistas na última década, como a independência financeira e inclusive a liberdade para frequentar ambientes onde se consomem bebidas alcoólicas; antes frequentado na sua maioria por homens.

A percepção que o adolescente tem sobre os problemas decorrentes do consumo de álcool não acompanha, necessariamente, a gravidade das consequências negativas. Os resultados encontrados no presente estudo evidenciam que as consequências mais relatadas pelos adolescentes em relação ao consumo de álcool foram a incapacidade de se lembrar de algo após o consumo e o sentimento de culpa ou remorso após ter bebido. Percebe-se ainda pouca preocupação por parte de familiares com relação ao consumo de álcool e seus efeitos na vida do adolescente. Isto talvez possa se justificar por se tratar de uma droga lícita e socialmente consumida pelos que cuidam destes adolescentes (POZZA et al. 2011).

$\mathrm{O}$ fato dos pais consumirem bebidas alcoólicas representou, no presente estudo, outro preditor do consumo de álcool entre os adolescentes. Vários estudos têm demonstrado o papel dos fatores familiares no consumo excessivo de álcool e drogas ilícitas (JORGE et al., 2017; SANCHEZ et al., 2011).

$\mathrm{O}$ adolescente mesmo sem um diagnóstico de abuso ou dependência de álcool, pode ser prejudicado pelo consumo do álcool, à medida que se sente mais seguro para resolver situações ou tomarem iniciativa apenas sob efeito de bebidas alcoólicas (VINER e TAYLOR et al. 2007). No presente estudo $21,6 \%$ dos adolescentes foram classificados quanto ao risco do consumo de álcool em "uso de risco a possível dependência". Esta prevalência representa um dado importante a ser considerado em estudos futuros de intervenção.

O início do consumo de álcool mostrouse aumentar de acordo com a faixa etária do adolescente. No entanto, muitos adolescentes iniciam o consumo de álcool em idade precoce (MALTA et al., 2014), até mesmo antes dos 12 anos de idade, (MAGGS et al., 2015), constituindo-se um fator de risco para os padrões mais perigosos de consumo de álcool na adolescência.

No presente estudo, a maior frequência de participação em eventos religiosos esteve negativamente associada ao consumo de álcool pelos adolescentes. No entanto, houve uma predominância do consumo de álcool pelos adolescentes que disseram ser espíritas ou católicos. Acredita-se que este resultado se dá pela maior permissibilidade dentro da doutrina se comparado com outras religiões como, por exemplo, a evangélica. Estudos científicos demonstram que a religiosidade, expressa pelas idas frequentes à igreja e pela importância dada à religião professada têm apresentado uma associação com o menor consumo de álcool e uso de outras drogas 
(MILLER et al., 2000; DALGALARRONDO et al., 2004; BEZERRA et al., 2009).

Com relação às limitações, primeiro, este estudo apresenta possível viés de memória. Em contrapartida, instrumentos auto-aplicáveis costumam deixar os participantes mais à vontade para responder questões, especialmente as consideradas sigilosas. Segundo, como o desenho de estudo foi transversal, outra limitação é a impossibilidade de se estabelecer causalidade. Terceiro, os resultados não podem ser extrapolados para a totalidade da população de adolescentes de Belo Horizonte.

Desta forma, torna-se de extrema relevância que estudos longitudinais sejam realizados de forma a possibilitar análises mais específicas quanto aos padrões de consumo, avaliações do risco e dependência do álcool, bem como os possíveis problemas e consequências em longo prazo.

\section{CONCLUSÃO}

O presente estudo mostrou uma alta prevalência do consumo de álcool entre os participantes. As consequências relacionadas ao uso do álcool mais relatadas foram a incapacidade de lembrar algo após o consumo de bebida alcoólica e o sentimento de culpa ou remorso por ter bebido. Houve associação estatisticamente significativa entre o consumo de bebidas alcoólicas com adolescentes de melhores condições socioeconômicas, filhos de pais que fazem o uso do álcool e que frequentam com menor frequência a igreja. $\mathrm{O}$ sexo não foi estatisticamente associado ao consumo de bebidas alcoólicas.

\section{REFERÊNCIAS}

BARTKOWSKI J.P., XU X. Religiosity and teen drug use reconsidered: a capital perspective. Am J Prev Med 2007; 32:182194.

BAUS J.; KUPEK E.; PIRES M. Prevalência e fatores de risco relacionados ao uso de drogas entre escolares. Rev Saude Publica 2002; 36:40-46.

BEZERRA J., BARROS, M.V.G.; TENÓRIO M.C.M.; TASSITANO R.M.; BARROS S.S.H.; HALLAL P.C. Religiosidade, consumo de bebidas alcoólicas e tabagismo em adolescentes. Rev Panam Salud Publica. 2009; 26:440-446.

CASTRO D.S.; SANCHEZ Z.M.; ZALESKI M.; ALVES H.N.; PINSKY I.; CAETANO R.; et al. Sociodemographic characteristics associated with binge drinking among Brazilians. Drug Alcohol Depend 2012; 126:272-276.

DALGALARRONDO P.; SOLDERA M. A.; FILHO H.R.C.; SILVA C.A.M. Religião e uso de drogas por adolescentes. Rev Bras Psiquiatr 2004; 26: 82-90.

DELMA P.O.S.; KELSY N.A.; DARTIU X.S.F. Alcohol and alcoholism among Brazilian adolescent public-school students. Revista saúde pública 2005; 39:585-92.

EL-MAAYTAH M.; SMITH S.F.; JERJES W.; UPILE T.; PETRIE A.; KALAVREZOS N.; et al. The effect of the new " 24 hour licensing law" on the incidence of facial trauma in London. Br J Oral Maxillofac Surg 2008; 46:460-463.

GUIMARÃES J.L.; GODINHO P.H.; CRUZ R.; KAPPANN J.I.; TOSTA J.L.A. Consumo de drogas psicoativas nos adolescentes escolares de Assis, SP. Rev Saude Publica 2004; 38:130-132. 
HUMENSKY J.L. Are adolescents with high socioeconomic status more likely to engage in alcohol and illicit drug use in early adulthood? Subst Abuse Treat Prev Policy 2010; 5:19.

JORGE K.O.; FERREIRA R.C.; FERREIRA E.F.; VALE M.P.; KAWACHI I.; ZARZAR P.M. Binge drinking and associated factors among adolescents in a city in southeastern Brazil: a longitudinal study. Cad Saude Publica 2017; 33:e00183115.

LAVERICK S.; PATEL N.; JONES D.C. Maxillofacial trauma and the role of alcohol. Br. J. Oral Maxillofac Surg 2008; 46:542-546.

LIMA C.T.; FREIRE A.C.; SILVA A.P.; TEIXEIRA R.M.; FARREL M.; PRINCE M. Concurrent and construct validity of the AUDIT in an urban Brazilian sample. Alcohol and Alcoholism 2005; 40:584-589.

MAGGS J.L.; STAFF J.; PATRICK M.E.; WRAY-LAKE L.; SCHULENBERG J.E. Alcohol Use at the Cusp of Adolescence: A Prospective National Birth Cohort Study of Prevalence and Risk Factors. J Adolesc Health 2015; 56: 639-645.

MAGNABOSCO M.B.; FORMIGONI M.L.; RONZANI T.M. Avaliação dos padrões de uso de álcool em usuários de serviços de atenção primária à saúde de Juiz de Fora e Rio Pomba (MG). Rev bras epidemiol 2007; 40:637-647.

MALDONADO-DEVINCCI A.M.; BADANICH K.A.; KIRSTEIN C.L. Alcohol during adolescence selectively alters immediate and long-term behavior and neurochemistry.

Alcohol 2010; 44:57-66.

MALTA D.C.; MACHADO I.E.; PORTO D.L.; SILVA M.M.A.; FREITAS P.C.; COSTA A.W.N.; OLIVEIRA-CAMPOS M. Alcohol consumption among Brazilian Adolescents according to the National Adolescent Schoolbased Health Survey (PeNSE 2012). Revista Bras Epidemiol SUPPL PeNSE 2014; 203214.

MARTINS-OLIVEIRA J.G.; JORGE K.O.; FERREIRA R.C.; FERREIRA E.F.; VALE M.P.; ZARZAR P.M. Risk of alcohol dependence: prevalence, related problems and socioeconomic factors. Cien Saude Cole 2016; 21:17-26.

MILLER L.; DAVIES M.; GREENWALD S. Religiosity and substance use and abuse among adolescents in the national comorbidity survey. J Am Acad Child Adolesc Psychiatry 2000; 39:1190-1197.

PAIVA P.C.P.; PAIVA H.N.; FILHO P.M.O.; LAMOUNIER J.A.; FERREIRA R.C.;

FERREIRA E.F.; ZARZAR P.M. Prevalence of traumatic dental injuries and its association with binge drinking among 12-year-olds: a population-based study. Int J Paediatr Dent 2015; 25:239-247.

POZZA A.M.; FONSECA G.A.A.; LACERDA G.A.F.; FARIAS A.A. A influência familiar no envolvimento dos jovens com as drogas.

Revista Eletrônica da Univar 2011; 7:154 157.

ROMANO M.; DUAILIBI S.; PINSKY I.; LARANJEIRA R. Alcohol purchase survey by adolescents in two cities of State of São Paulo, Southeastern Brazil. Rev Saude Publica 2007; 41:495-501.

SANCHEZ Z.M.; MARTINS S.S.; OPALEYE E.S.; MOURA Y.G.; NOTO A.R. Social factors associated to binge drinking: a cross-sectional survey among Brazilian students in private high schools. BMC Public Health 2011; 11:201.

TAVARES B.F.; BÉRIA J.U.; LIMA M.S. Drug use prevalence and school performance among teenagers. Rev Saude Publica, 2001; 35:150-158.

VINER R.M.; TAYLOR B. Adult outcomes of binge drinking in adolescence: findings from a UK national birth cohort. J Epidemiol Community Health 2007; 61:902- 907.

ZARZAR P.M.; JORGE K.O.; OKSANEN T.; VALE M.P.; FERREIRA E.F.; KAWACHI I. Association between binge drinking, type of friends and gender: A cross-sectional study among Brazilian adolescents. BMC Public Health 2012; 12:257. 